\title{
Trastornos de la conducta alimentaria y consumo de drogas en población adolescente
}

\section{Eating Disorders and drug use in adolescents}

\author{
| David Bisetto Pons*, ${ }^{* * *}$; Álvaro Botella GuiJarro*; \\ Alberto SanCho Muñoz ${ }^{* *}$
}

$\mid \begin{aligned} & \text { *Fundación para el Análisis, Estudio y Prevención de las Adicciones (AEPA). } \\ & { }^{\star \star} \text { Unidad de Conductas Adictivas de Xàtiva. Dpto. } 14 . \\ & { }^{\star \star \star} \text { Facultad de Psicologia. Universitat de València. }\end{aligned}$

Enviar correspondencia a:
David Bisetto Pons
Centro de Día AEPA
C/ José Dolz
46600 Alzira (Valencia)

recibido: Febrero 2011 aceptado: Septiembre 2011

\section{Resumen}

El estudio tuvo como objetivos demostrar si existía relación entre el consumo de drogas y los trastornos de conducta alimentaria, asi como qué tipo de drogas son las más utilizadas y si estas se utilizan para suprimir el apetito.

Se desarrolló una escala "ad hoc" formada por los items de la Eating Disorder Diagnostic Scale, cuyo objeto es detectar los casos de riesgo de padecer algún tipo de Trastorno de la Conducta Alimentaria, y por ítems cuyo objeto era evaluar el consumo de drogas en una muestra de 446 adolescentes estudiantes de $1^{\circ}$ de la enseñanza secundaria obligatoria a $2^{\circ}$ de Bachillerato de distintos Institutos de Educación Secundaria de la Comunidad Valenciana, con edades comprendidas entre los 13 y 18 años de edad.

Se encontró una relación entre los adolescentes que consumian drogas y el encontrarse en algunos de los umbrales de riesgo de la escala utilizada $\left(\chi^{2}(1, N=444)=3.87 ; p=.049\right)$ no habiendo relación estadisticamente significativa con la variable "tipo de droga" (Alcohol, estimulantes, tabaco $u$ otras). No obstante se aprecia una relación significativa entre la variable "utilizar algún tipo de droga como supresora del apetito" y estar en riesgo de padecer un Trastorno de la Conducta Alimentaria siendo el tabaco la droga más utilizada (66\% de los englobados en el umbral de riesgo de la Eating Disorder Diagnotic Scale).

Se concluyó que los adolescentes de la muestra analizada que se engloban en alguno de los umbrales de riesgo consumen más drogas que los adolescentes que no se engloban en dicho umbral de la Eating Disorder Diagnostic Scale, siendo las drogas de tipo estimulante las más utilizadas por estos adolescentes con el objeto de suprimir el apetito.

Palabras clave: adolescentes, estudiantes, uso de sustancias, trastornos de la conducta alimentaria.

\begin{abstract}
The aim of the study was to show whether there was a connection between drug use and Eating Disorders, as well as to identify the type of drugs most widely used and to ascertain whether they are used to suppress appetite.

An "ad hoc" scale was developed using the items of the Eating Disorder Diagnostic Scale, whose aim is to detect cases at risk of certain types of eating disorder, and items for assessing drug use. This scale was applied to samples of teenagers $(n=446)$ aged 13-18 from various secondary schools in the Valencia Region (Comunidad Valenciana) in Spain.

An association was found between teenagers that use drugs, and particularly between the variable "use of some kind of drug as an appetite suppressant," and being at risk of having an eating disorder. Tobacco was the drug most commonly used (accounting for 66\% of those within the risk threshold of the Eating Disorder Diagnostic Scale).

We conclude that those teenagers from the sample who fall within any of the risk thresholds consume more drugs than those who do not fall within the risk threshold of the Eating Disorder Diagnostic Scale. Stimulant-type drugs are those most widely used by these teenagers with the aim of suppressing appetite.
\end{abstract}

Key words: adolescents, students, substance use, eating disorders. 
L os principales Trastornos de la Conducta Alimentaria (TCA) según el DSM-IV-TR (APA, 2000) son la anorexia nerviosa y la bulimia nerviosa, con una prevalencia en población adolescente española que puede oscilar entre el 0,11 y $0,64 \%$ para la anorexia nerviosa y del 1,24 al 2,29\% para la bulimia nerviosa (Peláez, 2003; Peláez, Labrador y Raich, 2004). Ambos tienen en común la preocupación excesiva por la silueta y por el peso corporal, diferenciándose en que las personas con bulimia nerviosa mantienen el peso igual o un poco por encima de la población general, cosa que con la anorexia nerviosa no sucede.

El DSM-IV-TR distingue una tercera categoría dentro de los trastornos de la conducta alimentaria que Ilama trastorno de la conducta alimentaria no especificado. El trastorno que va tomando una mayor relevancia, dentro de los TCA no especificados, es el trastorno por atracón. Este trastorno se desarrolla en uno de los apéndices del DSM-IV-TR, en el apartado dedicado a criterios y ejes para estudios posteriores, donde lo define como episodios recurrentes de atracones en ausencia de conductas compensatorias inapropiadas características de la bulimia nerviosa. La prevalencia para el trastorno por atracón oscila entre el 2 y el $8 \%$ (Borra, 2008; Fisher, Golden, Katzman y Kreipe, 1995).

Estos trastornos, presentan una alta comorbilidad con el uso/abuso de drogas (Bulik, Klump, Thorton, Kaplan, Devlin, Fichter et al., 2004; Castro-Fornieles, Díaz, Goti, Calvo, González, Serrano et al., 2010; Kurg, Treasure, Anderluh, Bellodi, Cellini, Di Bernardo et al., 2008) y las mujeres que padecen bulimia nerviosa suelen tener un ratio de uso/abuso de drogas mayor que la población general (Bushnell, Wells, McKenzie, Hornblow, Oakley-Browne y Joyce, 1994), mientras que algunos estudios afirman que entre las mujeres que padecen anorexia nerviosa, el uso o abuso de drogas no suele ser distinto del que se da en la población general (Holderness, Brooks, Gunn y Warren, 1994). Existen otros estudios que muestran un mayor riesgo de consumo de drogas ilegales en general entre las mujeres con anorexia nerviosa (AN) que en las que padecen bulimia nerviosa (BN), las que a lo largo de su historia vital han sido diagnosticadas de anorexia nerviosa y bulimia nerviosa (AN/BN), las que presentan trastorno por atracón (TA) o un grupo de control sin TCA, mientras que para el uso/abuso de alcohol era mayor el riego para los grupos de BN y AN/BN respecto a los grupos de AN, TA y control (Root, Pisetsky, Thornton, Lichtenstein, Pedersen y Bulik, 2010).

Parece relevante distinguir entre los diferentes subgrupos de TCA y los diferentes tipos de sustancias para concretar en que tipo de TCA se produce un mayor uso o abuso de drogas y de qué drogas. Sin embargo, son escasos los estudios que detallan el tipo de sustancia de uso o abuso que suele utilizar la población adolescente que cumple o que está en riesgo de cumplir alguno de los criterios diagnósticos del DSM-IVTR para los TCA, y los pocos que existen, han mostrado una relación directa entre las dietas y los atracones (con o sin conductas purgativas) con el uso o abuso de drogas estimulantes, como pueden ser: tabaco, anfetaminas, cocaína o medicación psicotrópica (Piran y Robinson, 2006; Stice y Shaw, 2003). Algunos estudios han encontrado que los pacientes con AN de tipo restrictivo, es decir, con ausencia de atracones o purgas, consumen menos cannabis y estimulantes que los de AN tipo compulsivo/purgativo, que se da cuando el individuo recurre regularmente a purgas $u$ otras conductas compensatorias (Root, Pinheiro, Thornton, Strober, Fernández-Aranda, Brant, et al., 2010; Stock, Goldberg, Corbett y Katzman, 2002). Otros estudios han puesto de manifiesto la presencia en los TCA de variables muy relacionadas con las conductas adictivas como es la impulsividad o depresión (Courbasson y Brunshaw, 2009; Stice, Burton y Shaw, 2004; Wonderlich, Connolly y Stice 2004). También se ha estudiado la mayor presencia de episodios de consumo de grandes cantidades de bebida (binge) en mujeres en riesgo de padecer TCA (Khaylis, Trockel y Barr, 2009).

Por otra parte, como señalan Castro-Fornieles y colaboradores (2010), los problemas del abuso de sustancias en adolescentes con trastornos de la conducta alimentaria, correlacionan con mayor número de problemas escolares, sociales y familiares que los que, padeciendo un TCA, no presentan consumo de sustancias, por lo que sería conveniente que se detectasen y se pueda tener un enfoque terapéutico específico, adecuado a estas personas.

Tanto estos últimos autores, como otros de los mencionados anteriormente (Courbasson et al., 2009; Piran et al., 2006; Khaylis et al., 2009; Root, et al., 2010) destacan las implicaciones terapéuticas y de prevención que el conocimiento de todas estas relaciones entre consumo de drogas y TCA tienen, tanto para el tratamiento y prevención de los TCA, como para el tratamiento y prevención de los usos o abusos de sustancias psicoactivas.

Siguiendo la línea marcada en los resultados y las propuestas de estos estudios, los objetivos que persigue la presente investigación son: (1) esclarecer si existe relación entre el consumo de drogas y los TCA en población adolescente española, y (2) en el caso de que exista relación, qué tipo de drogas son las más utilizadas y en qué medida esas drogas se utilizan para suprimir el apetito.

\section{Método}

\section{Tipo de estudio}

Estudio ex post facto, retrospectivo con muestra no probabilística, propositiva (Montero y León, 2007).

\section{Participantes}

Participaron 446 adolescentes con edades comprendidas entre los 13 y los 18 años, con un promedio de 15 años y una desviación típica (DT) de 1.38, de la Comunidad Valenciana. Se descartaron dos cuestionarios por considerarlos "no válidos", por lo que la muestra definitiva fue de 444 adolescentes, 179 hombres $(40.1 \%)$ y 265 mujeres $(59.4 \%)$. 
Los participantes se obtuvieron de cuatro Institutos de Educación Secundaria (IES), dos de ellos concertados y dos de carácter público.

En cada IES se seleccionó al azar una clase por curso (desde $1^{\circ}$ ESO hasta $2^{\circ}$ de Bachillerato) participando en el estudio todos los alumnos/as de la clase.

\section{Instrumento}

Se diseño un cuestionario "ad hoc" que comprendia los 22 items del Eating Disorder Diagnostic Scale(EDDS) (Stice, Fisher y Martínez, 2004; Stice, Telch y Rezvi, 2000) añadiendo cuatro ítems con preguntas referentes al consumo de drogas.

La Eating Disorder Diagnostic Scale (EDDS) (Stice et al., 2000) está compuesta por 22 items y su objetivo es evaluar los síntomas o criterios diagnósticos del DSM-IV-TR de Ios TCA anorexia nerviosa y bulimia nerviosa, tanto del tipo restrictivo como purgativo, clasificando a los sujetos en distintos umbrales dependiendo de si cumplen o no los criterios diagnósticos del DSM-IV-TR para los TCA. Además, hace lo mismo con el llamado trastorno por atracón, trastorno que se contemplará con mucha probabilidad en futuras ediciones del DSM y que actualmente está incluido con el mismo nombre en el DSM-IV-TR pero en la categoría de: "trastornos alimentarios no especificados".

Así pues, la escala EDDS (Stice et al., 2000) ofrece dos puntos de corte o umbrales para cada trastorno que permiten diferenciar casos que cumplirian los criterios diagnósticos (umbral máximo) del DSM-IV-TR para los TCA y casos denominados de "riesgo" (subumbral) que cumplen alguno de los criterios diagnósticos del DSM-IV-TR.

A partir de estos umbrales, la escala, y más concretamente, el algoritmo de corrección proporcionado por los autores (Stice et al., 2004) permite clasificar a los sujetos en siete subcategorías dependiendo del riego de presentar un trastorno de la conducta alimentaria: (1) umbral completo de anorexia nerviosa, (2) umbral completo de bulimia nerviosa, (3) umbral completo de trastorno por atracón, (4) subumbral de anorexia nerviosa, que correspondería a la anorexia nerviosa sin amenorrea y con indice de masa corporal entre 17.5 y $18.5,(5)$ subumbral de bulimia, que correspondería a bulimia nerviosa, con la práctica de una conducta compensatoria a la semana y sin excesiva preocupación por el peso y la figura, (6) subumbral de trastorno por atracón, en el que la frecuencia de los episodios de atracón sería de uno por semana, y (7) una última categoría llamada de "riesgo inexistente" en la que se situarían los sujetos que no cumplen ningún criterio del DSM-IV-TR para los TCA.

\section{Procedimiento}

Se obtuvieron los permisos pertinentes de los centros educativos para poder administrar los cuestionarios a la muestra escogida y se informó a los centros, y éstos a los padres, de los fines del estudio, del carácter voluntario de la participación, de que la colaboración consistía en completar el cuestionario mencionado, de que éste era anónimo (de hecho no recogía ningún dato que pueda identificar directamente al participante) y de que se garantizaba la confidencialidad y el anonimato. También se informaba de todo esto a los participantes antes de administrar los cuestionarios.

La muestra seleccionada completó el cuestionario autoadministrado en presencia del psicopedagogo/a del centro educativo o del técnico de la Unidad de Prevención Comunitaria (UPC), entrenados para dicha tarea, siendo el tiempo medio para la contestación del EDDS (Stice et al., 2004) y los ítems añadidos de 25 minutos. Esta fase de cumplimentación de la escala se realizó durante los meses de octubre y noviembre de 2009.

Una vez concluido el proceso anterior, desde el centro educativo se dio aviso telefónico al equipo investigador para recogerlos o bien enviarlos por correo ordinario.

\section{Análisis de los datos}

Recogidos todos los cuestionarios, se realizó la codificación de estos para su posterior análisis con el programa estadístico SPSS-15.0 para Windows.

Un paso previo a dicha codificación, fue el descartar los cuestionarios denominados "no válidos" (aquellos que faltaba algún ítem por contestar o bien porqué faltaba algún tipo de información relevante) pasando luego a la corrección de los cuestionarios denominados "válidos" (aquellos que si cumplían los criterios mínimos).

Para la corrección se utilizó la sintaxis para SPSS que los propios autores facilitan en uno de sus artículos (Stice et al., 2004).

\section{Medidas y variables}

Se han utilizado tres variables para medir el riesgo de presentar un TCA. Estas variables se diferencian por el número de categorías en las que se clasifican los sujetos que están en riesgo, añadiendo siempre una categoría que recoge a los sujetos "sin riesgo".

Yendo de mayor a menor número de categorias, la primera de estas tres variables recoge las siete categorias originales de la EDDS descritas anteriormente. Esta variable se utilizó para describir la prevalencia en la muestra en cada grupo de riesgo.

La segunda variable se creó, a partir de estas siete categorías, agrupando a los individuos según el trastorno para el que estaban en riesgo (anorexia nerviosa, bulimia nerviosa y atracones). Así pues, para las personas consideradas de "riesgo" (casos dentro de las categorias 1 a 6) se establecieron tres categorías, una para cada trastorno: anorexia (categorías 1 y 4), bulimia (categorias 2 y 5) y atracones (categorias 3 y 6), y se añadió una cuarta categoría con los sujetos clasificados como de "riesgo inexistente" (categoría 7). Esta variable 
se utilizó para estudiar la relación entre riesgo de sufrir cada uno de los posibles TCA y consumo de drogas

La tercera variable, partiendo de la categorización de la EDDS, aglutinaba la muestra estudiada en dos categorías: (1) los que tenian "riesgo de presentar algún tipo de trastorno de la conducta alimentaria" (categorias de la 1 a la 6), y (2) los sujetos de "riesgo inexistente" (categoría 7). Se utilizó esta variable para estudiar la relación entre riesgo de presentar un TCA y consumo de drogas.

El consumo de drogas fue valorado añadiendo cuatro items. El primero permitía distinguir entre los que consumían drogas de los que no consumian (variable categórica con dos valores), en el caso de consumir algún tipo de droga, el segundo ítem preguntaba qué tipo de drogas eran las que se consumian y el tercer item preguntaba si el uso de esas drogas tenía como fin el suprimir el apetito (variable categórica con dos valores). En el caso de ser la respuesta afirmativa en este ítem, se pasaba al último ítem que les preguntaba qué tipo de drogas utilizaban para conseguir dicho fin.

El consumo de drogas se refería al uso de drogas al menos una vez por semana.

Con respecto a los ítems que preguntaban qué drogas se consumian, se permitían cinco alternativas de elección múltiple pudiendo optar entre: Alcohol, Tabaco, Cannabis, Estimulantes y Otras. Así pues, el sujeto podia elegir una o varias alternativas dependiendo del tipo de drogas que consumía. En la quinta opción de respuesta ("otras") el sujeto podria indicar otro tipo de sustancias no reflejadas en el ítem. Se distinguió entre la opción "Tabaco" y la opción "Estimulantes (cocaína, anfetamina, etc.)", aún teniendo ambas en común el

Tabla 1. Variables utilizadas y sus categorías

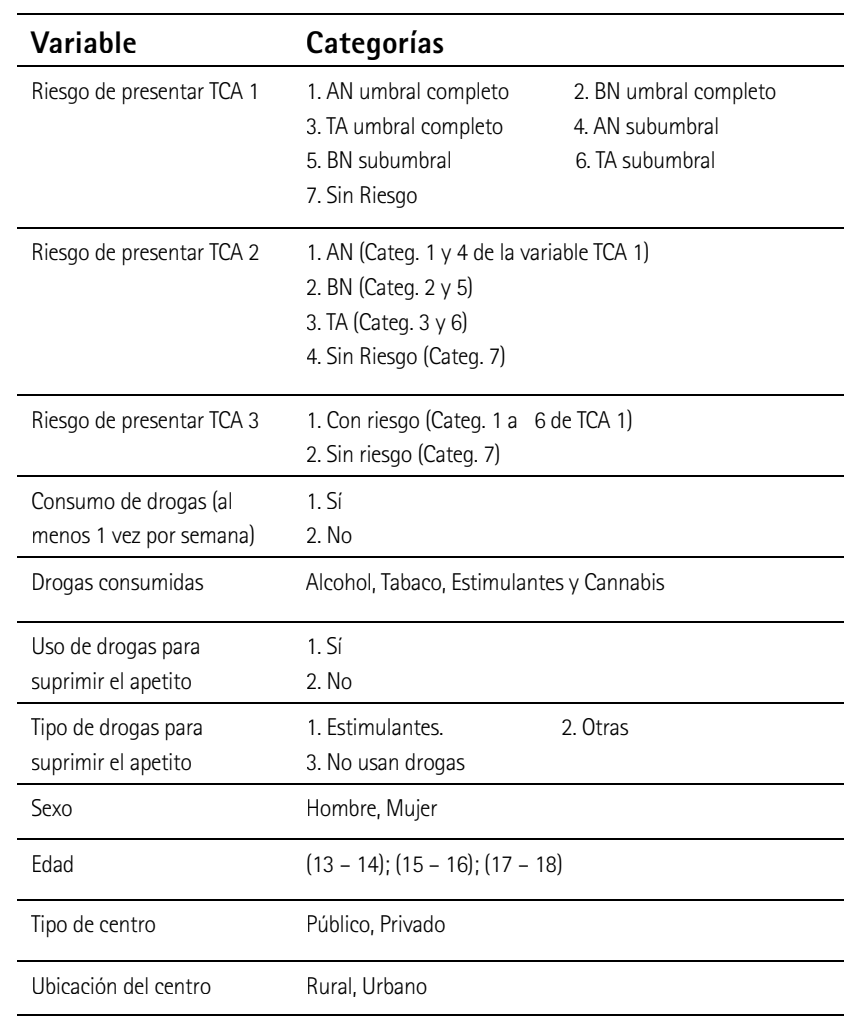

ser estimulantes del Sistema Nervioso Central (SNC), con el objeto de facilitar la comprensión de los adolescentes y además por ser el tabaco una de las drogas de uso o abuso más consumida entre los mismos (ESTUDES, 2008).

Con el objeto de facilitar el análisis de los datos obtenidos con respecto al consumo de drogas para suprimir el apetito, se creó una variable con tres categorias: una en la que se agrupó en una misma categoría el uso/abuso de estimulantes y el uso/abuso de tabaco, ya que ambas tienen efectos estimulantes sobre el sistema nervioso central (SNC); otra en la que se agrupó el uso/abuso de las demás drogas y una tercera categoría en la que se incluyó a los adolescentes que no consumian drogas.

Otras variables que se han tenido en cuenta en el presente estudio, han sido: tipo de centro educativo (público - concertado) y ubicación del centro (rural - urbano).

El resto de las variables analizadas son el sexo y la edad, esta última agrupándose en tres categorías o intervalos: (13 - 14) años, (15 - 16) años y (17 - 18) años.

En la Tabla 1 se resumen las variables utilizadas.

\section{Resultados}

En cuanto a la variable riesgo de padecer un TCA 3, existe una relación estadísticamente significativa en la muestra analizada con la variable sexo $\left[\chi^{2}(1, N=444)=2.34 ; p<\right.$ .001 ; Odds - Ratio $(\mathrm{OR})=2.55$, con un intervalo de confianza IC para la OR, IC $(95 \%)=(1.49,4.34)]$. En porcentajes, obtenemos un $11.7 \%$ de los hombres está en riesgo, frente a un $25.28 \%$ de las mujeres.

No se ha encontrado relación estadísticamente significativa entre la variable uso de drogas y la variable sexo $\left[\chi^{2}(1, N\right.$ $=444)=0.58 ; \mathrm{p}=.45 ; \mathrm{OR}=0.85 ; \mathrm{IC}(95 \%)=(0.56,1.29)]$.

En cuanto a los grupos de edad establecidos, no parece existir relación estadísticamente significativa entre estar en riesgo de padecer un TCA 3 y el grupo de edad al que se pertenece $\left(\chi^{2}(2, N=444)=1.78 ; p=.41\right)$, aunque la tendencia es a ser ligeramente más elevada en el rango de edad de (17 - 18) años, con un $25.33 \%$ de personas en riesgo, frente al $19.15 \%$ del rango de $(13$ - 14) años, o frente el $18.23 \%$ del rango de $(15-16)$ años.

No obstante, si que se encuentra relación estadisticamente significativa entre grupo de edad y consumo de drogas $\left(\chi^{2}(2, N=444)=2.98 ; p<.001\right)$. Así, entre los sujetos analizados dicen consumir algún tipo de droga un $19.1 \%$ en el rango de edad comprendido entre (13 - 14) años, un 36.5\% en el rango de $(15$ - 16) años y un $48 \%$ en el rango de (17 18) años.

Algo similar ocurre con las variables: tipo de centro educativo (público frente a concertado) y tipo de ubicación (urbano frente a rural). Asi pues, para el tipo de centro, no 
hay asociación estadísticamente significativa con la variable: riesgo de padecer TCA $3\left(\chi^{2}(1, N=444)=0.15 ; p=.67\right)$ y sí parece existir con el consumo de drogas $\left[\chi^{2}(1, N=444)=\right.$ $6.72 ; p=.01 ; O R=1.96$, IC $(95 \%)=(1.17,3.26)]$. En porcentajes, un $34.3 \%$ de los alumnos en centro público consumen algún tipo de drogas, frente al $21.1 \%$ de los alumnos en centros concertados.

Con respecto a la variable tipo de ubicación (urbano o rural), no hay asociación estadísticamente significativa con la variable riesgo de padecer un TCA $3\left(\chi^{2}(1, N=444)=0.45\right.$; $\mathrm{p}=.50)$ y sí la hay con la variable consumo de drogas $\left[\chi^{2}(1\right.$, $N=444)=19.60 ; p<.001 ; O R=2.81 ;$ IC $(95 \%)=(1.76$, 4.49)]. En porcentajes, $38.4 \%$ de los alumnos en centro en ámbito rural consumen algún tipo de droga, mientras que en los centros de ámbito urbano lo hacen el $18.1 \%$.

La prevalencia en las siete categorias de riesgo de padecer un TCA detectadas por la escala EDDS (Stice et al., 2004) se muestran en la Tabla 2.

Tabla 2. Prevalencia, por sexo y total, en la muestra del riesgo de presentar un TCA, según la EDDS, casos y porcentajes

\begin{tabular}{|c|c|c|c|c|c|c|c|}
\hline \multirow{2}{*}{\multicolumn{2}{|c|}{ Riesgo de presentar TCA 1}} & \multicolumn{2}{|c|}{ Hombres } & \multicolumn{2}{|c|}{ Mujeres } & \multicolumn{2}{|c|}{ Total } \\
\hline & & Casos & $\%$ & Casos & $\%$ & Casos & $\%$ \\
\hline Sin riesgo & & 158 & 88.3 & 198 & 74.7 & 356 & 80.2 \\
\hline \multirow{3}{*}{ Umbral Completo } & Anorexia & 0 & 0 & 3 & 1.1 & 3 & 0.7 \\
\hline & Bulimia & 4 & 2.2 & 15 & 5.7 & 19 & 4.3 \\
\hline & Atracones & 0 & 0 & 4 & 1.5 & 4 & 0.9 \\
\hline \multirow{3}{*}{ Subumbral } & Anorexia bajo & 0 & 0 & 7 & 2.6 & 7 & 1.6 \\
\hline & Bulima bajo & 16 & 8.9 & 34 & 12.8 & 50 & 11.3 \\
\hline & $\begin{array}{l}\text { Atracones } \\
\text { bajo }\end{array}$ & 1 & 0.6 & 4 & 1.5 & 5 & 1.1 \\
\hline \multicolumn{2}{|l|}{ Total } & 179 & 100.0 & 265 & 100.0 & 444 & 100.0 \\
\hline
\end{tabular}

En cuanto a la relación entre la variable riesgo de padecer TCA 3 y consumo de drogas, si que parecen estar asociadas en la muestra analizada $\left[\chi^{2}(1, N=444)=3.87 ; p=.049 ; 0 R\right.$ $=1.62 ; \mathrm{IC}(95 \%)=(1.08 ; 2.44)]$. Expresado en porcentajes, el $28.9 \%$ de los sujetos sin riesgo de padecer un TCA consumen alguna droga, mientras que, de los sujetos con riesgo de padecer un TCA, el porcentaje de los que toman alguna droga es $39.8 \%$.

Analizando por sexos esta misma relación, en el grupo de hombres parece existir una relación estadisticamente significativa entre riesgo de padecer TCA 3 y consumo de drogas $\left[\chi^{2}(1, N=179)=3.98 ; p=.046 ; O R=2.51 ; \operatorname{IC}(95 \%)=\right.$ $(0.99 ; 6.34)]$, tomando con mucha cautela esta significación estadística, dado que el límite inferior del intervalo de confianza para la odds ratio es menor que uno. En el grupo de mujeres no hay relación estadísticamente significativa entre riesgo de padecer TCA 3 y consumo de drogas $\left[\chi^{2}(1, N=265)\right.$ $=0.97 ; p=.326 ; O R=1.34 ;$ IC $(95 \%)=(0.75 ; 2.39)]$. Así, el $47.6 \%$ de los hombres en riesgo de presentar un TCA consu- me drogas, frente al 26.6\% que consume drogas en el grupo sin riesgo. En las mujeres, el 37.3\% del grupo en riesgo toma drogas, frente al $30.8 \%$ del grupo sin riesgo que lo hace.

En cuanto a la relación entre personas en riesgo de padecer un TCA 3 y consumo de drogas para suprimir el apetito, se da una asociación estadisticamente significativa $\left[\chi^{2}(1, N=\right.$ $444)=7.07 ; p<.01 ; O R=4.27 ;$ IC $(95 \%)=(1.34,13.57)]$. Así, el $6.8 \%$ de las personas con riesgo de padecer un TCA utilizan drogas para suprimir el apetito, frente al 1.7\% de los sujetos sin riesgo que también utilizan drogas para suprimir el apetito.

Analizando por sexos, no existe una relación estadísticamente significativa entre riesgo de padecer un TCA 3 y consumo de drogas para suprimir el apetito, ni en el grupo de hombres ni en el de mujeres. Para los hombres tenemos $\chi^{2}(1, N=176)=1.42 ; p=.233$, aplicando la discontinuidad de Yates, por tener casillas con frecuencias menores que 5. Para las mujeres, $\chi^{2}(1, N=263)=2.47 ; p=.0116 ; 0 R=$ 2.58 ; IC $(95 \%)=(0.76,8.75)$.

No hay relación estadisticamente significativa entre consumo de drogas y riesgo de padecer un TCA 2, es decir, cuando tenemos en cuenta los sujetos en riesgo para cada uno de los posibles trastornos (anorexia, bulimia y atracones) más la categoría de $\sin$ riesgo $\left(\chi^{2}(3, N=444)=4.07\right.$; $p=.254)$.

Las drogas que dicen consumir los que contestan al ítem, clasificadas por los grupos con y sin riesgo de padecer TCA, se muestran en la Tabla 3.

No hay relación entre el uso o no de cada tipo de droga y el riesgo o no de padecer un TCA 3. Los resultados de las pruebas estadísticas se muestran en la Tabla 4.

Tabla 3. Grupo que toma droga: Riesgo de TCA - Drogas consumidas (\%)

\begin{tabular}{ccccc}
\hline & Alcohol & Tabaco & Estimulantes & Cannabis \\
\hline Sin riesgo TCA & 92.2 & 30.1 & 5.8 & 25.2 \\
Con riesgo TCA & 82.9 & 37.1 & 8.6 & 22.9 \\
\hline
\end{tabular}

Tabla 4. Relación tipo de droga-estar en riesgo de presentar un TCA.

\begin{tabular}{lllll}
\hline & $\chi_{1}^{2}(1)$ & $p(2)$ & OR (3) & IC (4) \\
\hline Alcohol & 1.25 & .263 & 1.33 & $(0.81 ; 2.20)$ \\
Tabaco & 2.59 & .107 & 1.75 & $(0.88 ; 3.51)$ \\
Estimulantes & 1.06 & .304 & 2.06 & $(0.51 ; 8.40)$ \\
Cannabis & 0.22 & .639 & 1.22 & $(0.53 ; 2.78)$ \\
\hline
\end{tabular}

(1) Estadistico Chi-cuadrado, con un grado de libertad. (2) Nivel de significación. (3) Razón de ventajas (odds-ratio) del grupo con riesgo respecto al grupo sin riesgo. (4) Intervalo de Confianza al $95 \%$ para la OR. 
Existe relación estadísticamente significativa entre el uso o no de drogas estimulantes para suprimir el apetito y estar en riesgo de padecer un TCA $\left(\chi^{2}(2, N=444)=20.48\right.$; $p<.001)$, de tal manera que el $100 \%$ de los que usan drogas estimulantes para suprimir el apetito pertenecen al grupo de riesgo de padecer un TCA.

\section{Discusión}

Teniendo en cuenta los datos analizados, parece confirmarse en la muestra estudiada que existe relación estadísticamente significativa entre los adolescentes que tienen riesgo de sufrir un Trastorno de la Conducta Alimentaria y el consumo de drogas. Estos datos son similares a estudios previos realizados sobre la materia (Anzengruber, Klump, Thornton, Brandt, Crawford, Fichter, et al., 2006; Krug et al., 2008; Saules, Pomerleau, Snedecor, Mehringer, Shadle, Kurth, et al., 2004; Welch y Fairburn, 1998; Wiseman, Turco, Sunday y Halmi, 1998). Otra relación significativa se aprecia entre el riesgo de padecer un TCA y el consumo de drogas de carácter estimulante (sobre todo tabaco) con el objeto de suprimir el apetito o controlar el peso; este dato difiere del estudio de referencia utilizado para la presente investigación (Krug et al., 2008), pero coincide con otros estudios consultados (Austin y Gortmaker, 2001; Croll, Neumark-Sztainer, Story y Ireland, 2002; Delnevo, Hrywna, Abatemarco y Lewis, 2003; George y Waller, 2005).

Comparando los distintos TCA (bulimia nerviosa, anorexia nerviosa y el trastorno por atracón) con el consumo de drogas, parece no existir una relación significativa entre dichas variables. Este resultado no coincide con otras investigaciones en las que se aprecia una tendencia de los sujetos que presentan atracones a consumir más drogas (Corcos, Nezelof, Speranza, Topa, Girardon, Guilbaud, et al., 2001; McCabe y Boyd, 2005; Nappo, Tabach, Noto, Galduróz y Carlini, 2002; Piran et al., 2006). Dicho hallazgo puede deberse al tamaño de la muestra y a que se ha extraído de una población general no diagnosticada, de manera que hay muy pocos casos englobados en el umbral de riesgo de padecer un TCA.

El análisis de los datos realizados muestran otros resultados de interés como que el tipo de centro educativo (público - concertado) y zona dónde se ubica el mismo (urbano - rural), no parece tener relación con el estar en riesgo o no de padecer un TCA; no sucede lo mismo con la variable consumo de drogas y tipo de centro (público - concertado), ya que se ha encontrado mayor proporción de adolescentes de la muestra estudiada que consumen drogas (sobre todo tabaco) que provienen de centros públicos, dato que parece ir en contra de algunos estudios (Bjarnason, Davidaviciene, Miller, Nociar, Pavlakis y Stergar, 2003; Paniagua, Garcia, Castellano, Sarrallé y Redondo, 2001) pero a favor de otros (Yáñez, López, SerraBatlles, Roger, Arnau y Roura, 2006) no explicándose este hallazgo por un tamaño muestral insuficiente. De igual forma sucede con la variable ubicación del centro educativo (urbano - rural), apareciendo en este estudio una mayor proporción de alumnos/as que consumen drogas de centros ubicados en zona rural.
Con lo que respecta a los datos generales obtenidos sobre el consumo y tipo de droga, los datos obtenidos en la muestra estudiada no difieren de los resultados del informe del Plan Nacional sobre Drogas (ESTUDES, 2008) confirmando que las drogas que más utilizan los alumnos/as de enseñanza secundaria, por frecuencia de consumo o por importancia, son: el alcohol, tabaco y cannabis respectivamente.

Respecto a otras variables, como la variable sexo, los resultados obtenidos demuestran una vez más que los TCA se dan más en mujeres que en hombres, siendo la relación entre dichas variables significativa, no existiendo dicha diferencia en cuanto al consumo de drogas. Respecto a la variable edad, se ha obtenido una relación significativa entre ambas indicando la tendencia de que a mayor edad, mayor consumo de drogas.

\section{Implicaciones terapéuticas}

Los resultados enfatizan la importancia del consumo de drogas en adolescentes que cumplen todos o algún criterio para los TCA del DSM-IV-TR, aspecto que se debería de tener en cuenta a la hora de intervenir con dicha población, ya que si sólo se tratan los aspectos relacionados con el uso/abuso de sustancias o relacionados con cualquier trastorno psicológico (depresión, ansiedad, etc.) sin tener en cuenta dichos trastornos, estaremos abocados al fracaso terapéutico (Benjamin y Wulfert, 2005; Castro-Fornieles et al., 2010; Sinha y 0'Malley, 2000; Stice, Presnell y Bearman, 2001).

Así, se debería tener en cuenta los resultados obtenidos en esta y otras investigaciones en los distintos programas de prevención para la salud como son los de drogodependencias o los de la depresión, ansiedad, etc., que se aplican en los distintos centros educativos o los programas para la prevención de la depresión, etc. sobretodo si la población objeto de intervención contempla a población adolescente de sexo femenino, ya que, a nuestro entender, la prevención debe ser entendida como una intervención global y masiva en la que se deberia de reflejar aspectos tan importantes como el estilo de vida y, como no, la alimentación.

Otra fortaleza a resaltar de la presente investigación, es que el estudio ha contemplado datos de la posible incidencia de TCA y consumo de drogas en distintas zonas (rural vs. urbana), así como tipo de centro (concertado/público) de la muestra analizada abriendo así la puerta de futuras investigaciones en la materia.

\section{Limitaciones del estudio}

Los resultados obtenidos en el presente estudio deben ser considerados teniendo en cuenta una serie de importantes limitaciones como son: primero, el instrumento principal utilizado el EDDS (Stice et al., 2004) está en proceso de validación en población española; segundo, la selección de la muestra es no probabilística debiendo de ser cautos a la hora de extrapolar los resultados a otras poblaciones de adolescentes; tercero, el grupo considerado de riesgo a padecer un TCA presenta un número de casos insuficiente para sacar conclusiones generales del estudio. 


\section{Conflicto de intereses}

Los autores del artículo manifiestan no tener ningún conflicto de intereses.

\section{Referencias}

Anzengruber, D., Klump, K. L., Thornton, L., Brandt, H., Crawford, S., Fichter, M. M. y LaVia, M. (2006). Smoking in eating disorders. Eating Behaviors, 7, 91-299. doi:10.1016/j.physletb.2003.10.071

APA. (2000). Diagnostic and Statistical Manual of Mental Disorders. Washington, D.C.: Author.

Austin, S. B. y Gortmaker, S. L. (2001). Dieting and smoking initiation in early adolescent girls and boys: a prospective study. American Journal of Public Health, 91, 446-50.

Benjamin, L. y Wulfert, E. (2005). Dispositional correlates of addictive behaviors in college women: binge eating and heavy drinking. Eating Behaviors, 6, 197-209. doi:10.1016/j.eatbeh.2003.08.001

Bjarnason, T., Davidaviciene, A. G., Miller, P., Nociar, A., Pavlakis, A. y Stergar, E. (2003). Family structure and adolescent cigarette smoking in eleven European countries. Addiction, 98, 815-824. doi:10.1046/j.1360-0443.2003.00395.x

Borra, C. (2008). El atracón y sus trastornos. Caracteristicas psicopatológicas e implicaciones diagnósticas. Tesis doctoral. Universidad de Valencia. Recuperado el 15 de julio de 2010 de http://www.tdr.cesca.es/TESIS_UV/AVAILABLE/TDX-0917109160902/borra.pdf

Bulik, C. M., Klump, K. L., Thornton, L., Kaplan, A. S., Devlin, B., Fichter, M. M. y Crow, S. (2004). Alcohol use disorder comorbidity in eating disorders: A multicenter study. Journal of Clinical Psychiatry, 65, 1000-1006.

Bushnell, J. A., Wells, J., McKenzie, J., Hornblow, A., Oakley-Browne, M. y Joyce, P. (1994). Bulimia comorbidity in the general population and in the clinic. Psychological Medicine, 24, 605-611. doi: 10.1017/S0033291700027756

Castro-Fornieles, J., Diaz, R., Goti, J., Calvo, R., Gonzalez, L., Serrano, L. y Gual, A. (2010). Prev-alence and factors related to substance use among adolescents with eating disorders. European Addiction Research, 16, 61-68. doi:10.1159/000268106

Corcos, M., Nezelof, S., Speranza, M., Topa, S., Girardon, N., Guilbaud, 0. y Venisse, J. (2001). Psychoactive substance consumption in eating disorders. Eating Behaviors, 2, 27-38. doi:10.1016/S14710153(00)00021-0

Courbasson, C. y Brunshaw, J. M. (2009). The relationship between concurrent substance use dis-orders and eating disorders with personality disorders. International Journal of Environmental Research and Public Health, 6, 2076-2089. doi:10.3390/ ijerph6072076

Croll, J., Neumark-Sztainer, D., Story, M. y Ireland, M. (2002). Prevalence and risk and protective factors related to disordered eating behaviors among adolescents: Relationship to gender and ethnicity. Journal of Adolescent Health, 31, 166-175. doi:10.1016/ S1054-139X(02)00368-3

Delnevo, C. D., Hrywna, M., Abatemarco, D. J. y Lewis, M. J. (2003). Relationships between ciga-rette smoking and weight control in young women. Family \& Community Health, 26, 140-146.

Fisher, M., Golden, N. H., Katzman, D. K. y Kreipe, R. E. (1995). Eating disorders in adolescents: A background paper. Journal of Adolescent Health, 16, 420-437. doi:10.1016/1054139X(95)00069-5

George, A. y Waller, G. (2005). Motivators for smoking in women with eating disorders. European Eating Disorders Review, 13, 417-423. doi:10.1002/erv.623

Holderness, C. C., Brooks Gunn, J. y Warren, M. P. (1994). Comorbidity of eating disorders and substance abuse review of the literature. International Journal of Eating Disorders, 16, 1-34. doi:10.1002/1098-108X(199407)16:1<1::AIDEAT2260160102>3.0.CO;2-T

Khaylis, A., Trockel, M. y Taylor, C. B. (2009). Binge drinking in women at risk for developing eating disorders. International Journal of Eating Disorders, 42. 409-414. doi:10.1002/eat.20644

Krug, I., Treasure, J., Anderluh, M., Bellodi, L., Cellini, E., Di Bernardo, M. y Penelo, E. (2008). Present and lifetime comorbidity of tobacco, alcohol and drug use in eating disorders: A Euro-pean multicenter study. Drug and Alcohol Dependence, 97, 169-179. doi:10.1016/j. drugalcdep.2008.04.015

McCabe, S. E. y Boyd, C. J. (2005). Sources of prescription drugs for illicit use. Addictive Behaviors, 30, 1342-1350. doi:10.1016/j. addbeh.2005.01.012

Montero, I. y León, 0. G. (2007). A guide for naming research studies in Psychology. International Journal of Clinical and Health Psychology, 7, 847-862.

Nappo, S. A., Tabach, R., Noto, A. R., Galduróz, J. C. F. y Carlini, E. A. (2002). Use of anorectic amphetamine-like drugs by Brazilian women. Eating Behaviors, 3, 153-165. doi:10.1016/S14710153(01)00054-X

Paniagua, H., García, S., Castellano, G., Sarrallé, R. y Redondo, C. (2001). Consumo de tabaco, alcohol y drogas no legales entre adolescentes y la relación con los hábitos de vida del entorno. Anales Españoles de Pediatría, 55, 121-128.

Peláez, M. A. (2003). Estudio epidemiológico de los trastornos de la conducta alimentaria en población escolarizada de la Comunidad de Madrid. Tesis doctoral, Universidad Complutense. Madrid. Recuperado el 20 de octubre de 2010 de http://www.ucm.es/ BUCM/tesis/psi/ucm-t26738.pdf

Peláez, M. A., Labrador, F. J. y Raich, R. M. (2004). Epidemiología de los trastornos de la conducta alimentaria en España: Revisión y estado de la cuestión. Cuadernos de Medicina Psicosomática y Psiquiatria de Enlace, 71-72, 33-42.

Piran, N. y Robinson, S. R. (2006). The association between disordered eating and substance use and abuse in women: A communitybased investigation. Women \& Health, 44, 1-20. doi:10.1002/ eat. 10050

Plan Nacional sobre Drogas (2008). Informe de la encuesta estatal sobre uso de drogas en estudiantes de enseñanzas secundarias (ESTUDES) 2008. Ministerio de Sanidad y Política Social. Delegación del Gobierno para el Plan Nacional sobre Drogas (DGPNSD).

Root, T. L., Pinheiro, A. P., Thornton, L., Strober, M., Fernandez Aranda, F., Brandt, H. y Johnson, C. (2010). Substance use disorders in women with anorexia nervosa. International Journal of Eating Disorders, 43, 14-21. doi:10.1002/eat.20670

Root, T. L., Pisetsky, E. M., Thornton, L., Lichtenstein, P., Pedersen, N. L., y Bulik, C. M. (2010). Patterns of co-morbidity of eating disorders and substance use in Swedish females. Psychological Medicine, 40, 105-115. doi:10.1017/S0033291709005662

Saules, K. K., Pomerleau, C. S., Snedecor, S. M., Mehringer, A. M., Shadle, M. B., Kurth, C. y Krahn, D. D. (2004). Relationship of 
onset of cigarette smoking during college to alcohol use, dieting concerns, and depressed mood: Results from the young women's health survey. Addictive Behaviors, 29, 893-899. doi:10.1016/j. addbeh.2004.02.015

Sinha, R. y O'Malley, S. S. (2000). Alcohol and eating disorders: Implications for alcohol treatment and health services research. Alcoholism: Clinical and Experimental Research, 24, 1312-1319. doi:10.1111/j.1530-0277.2000.tb02097.x

Stice, E., Burton, E. M. y Shaw, H. (2004). Prospective relations between bulimic pathology, de-pression, and substance abuse: Unpacking comorbidity in adolescent girls. Journal of Consult-ing and Clinical Psychology, 72, 62-71. doi:10.1037/0022-006X.72.1.62

Stice, E., Fisher, M. y Martinez, E. (2004). Eating disorder diagnostic scale: Additional evidence of reliability and validity. Psychological Assessment, 16, 60-71. doi:10.1037/1040-3590.16.1.60

Stice, E., Presnell, K. y Bearman, S. K. (2001). Relation of early menarche to depression, eating disorders, substance abuse, and comorbid psychopathology among adolescent girls. Developmental Psychology, 37, 608-619. doi:10.1037/00121649.37.5.608

Stice, E. y Shaw, H. (2003). Prospective relations of body image, eating, and affective disturbances to smoking onset in adolescent girls: How Virginia slims. Journal of Consulting and Clinical Psychology, 71, 129-135. doi:10.1037/0022-006X.71.1.129

Stice, E., Telch, C. F. y Rizvi, S. L. (2000). Development and validation of the eating disorder di-agnostic scale: A brief self-report measure of anorexia, bulimia, and binge-eating disorder. Psychological Assessment, 12, 123-131. doi:10.1037/1040-3590.12.2.123

Stock, S. L., Goldberg, E., Corbett, S. y Katzman, D. K. (2002). Substance use in female adolescents with eating disorders. Journal of Adolescent Health, 31, 176-182.

Welch, S. L. y Fairburn, C. G. (1998). Smoking and bulimia nervosa. International Journal of Eating Disorders, 23, 433437. doi:10.1002/(SICI) 1098-108X(199805)23:4<433::AIDEAT11>3.0.CO;2-X

Wiseman, C. V., Turco, R. M., Sunday, S. R. y Halmi, K. A. (1998). Smoking and body image con-cerns in adolescent girls. International Journal of Eating Disorders, 24, 429-433. doi:10.1002/(SICI)1098108X(199812)24:4<429::AID-EAT10>3.0.C0;2-D

Wonderlich, S. A., Connolly, K. M. y Stice, E. (2004). Impulsivity as a risk factor for eating disor-der behavior: Assessment implications with adolescents. International Journal of Eating Disorders, 36, 172-182. doi:10.1002/eat.20033

Yáñez, A. M., López, R., Serra-Batlles, J., Roger, N., Arnau, A. y Roura, P. (2006). Consumo de tabaco en adolescentes. estudio poblacional sobre las influencias parentales y escolares. Archi-vos de Bronconeumología, 42, 21-24. 